J. Korean Math. Soc. 50 (2013), No. 6, pp. 1291-1310

http://dx.doi.org/10.4134/JKMS.2013.50.6.1291

\title{
PSEUDO-SPECTRAL LEAST-SQUARES METHOD FOR ELLIPTIC INTERFACE PROBLEMS
}

\author{
ByeOng-CHun SHIN
}

\begin{abstract}
This paper develops least-squares pseudo-spectral collocation methods for elliptic boundary value problems having interface conditions given by discontinuous coefficients and singular source term. From the discontinuities of coefficients and singular source term, we derive the interface conditions and then we impose such interface conditions to solution spaces. We define two types of discrete least-squares functionals summing discontinuous spectral norms of the residual equations over two sub-domains. In this paper, we show that the homogeneous least-squares functionals are equivalent to appropriate product norms and the proposed methods have the spectral convergence. Finally, we present some numerical results to provide evidences for analysis and spectral convergence of the proposed methods.
\end{abstract}

\section{Introduction}

Let $\Omega$ be the square $(-1,1)^{2}$ and let $\Omega_{1}=(-1,0) \times(-1,1)$ and $\Omega_{2}=$ $(0,1) \times(-1,1)$ be two open subsets of $\Omega$. Denote by $\Gamma$ the interface between $\Omega_{1}$ and $\Omega_{2}$, i.e., $\Gamma=\bar{\Omega}_{1} \cap \bar{\Omega}_{2}$. We consider the second-order elliptic boundary value problem:

$$
\left\{\begin{aligned}
-\nabla \cdot(a \nabla p)+\mathbf{b} \cdot \nabla p+c_{0} p & =f+\nu \delta(x) g(y) & & \text { in } \Omega, \\
p & =0 & & \text { on } \Gamma_{D}, \\
\mathbf{n} \cdot(a \nabla p) & =0 & & \text { on } \Gamma_{N},
\end{aligned}\right.
$$

where $\partial \Omega=\Gamma_{D} \cup \Gamma_{N}$ denotes the boundary of $\Omega, f$ is a given piecewise continuous function; $a$ and $c_{0}$ are piecewise constant, $\mathbf{b}$ is a piecewise vector constant, and $\mathbf{n}$ is the outward unit vector normal to the boundary. Here, $\nu \delta(x) g(y)$ is a singular source term where $\delta(x)$ denotes the Dirac $\delta$-function with the support on the interface $\Gamma, \nu$ is a constant and $g(y)$ is a continuous function.

Received December 17, 2012.

2010 Mathematics Subject Classification. Primary 65F10, 65F30.

Key words and phrases. Pseudo-spectral method, least-squares method, interface problem.

The paper was supported by Basic Science Research Program through the National Research Foundation of Korea (NRF) funded by the Ministry of Education, Science and Technology (2010-0009731). 
In various applications of physics, engineering and biological sciences, differential equations and partial differential equations with discontinuous coefficients and/or singular source terms have been commonly used. The singular source terms are also used for modeling extremely small-scale perturbations, such as the wave interaction with a singular defect in a disordered media, particle-flow interactions, particle-particle interactions, etc([7], [19], [29]).

In recent years there has been lots of interest in the use of first-order system least-squares method (FOSLS) for numerical approximations of elliptic partial differential equations, Stokes equations and Navier-Stokes equations. Introducing an extra physically meaningful variable $\mathbf{u}=a \nabla p$, the equation (1.1) above can be written as an equivalent first-order system of linear equations (see [9], [10] and [24]). For a use of finite element method, the least-squares approach was widely studied in [14], [15], [17], [5], [8], [9], [10] and [18], and a leastsquares method for the interface problem of Poisson equations was introduced in [1] and [13]. Least-squares approaches using spectral method were also studied in [20], [21], [22] and [25]. The least-squares methods have several benefits such that the resulting algebraic system is always symmetric positive definite and the methods can avoid LBB compatibility condition. For more details we refer to [6] and references therein.

For an interface problem without any singular source term, in [13] they proposed and analyzed a least-squares finite element method. To avoid global regularity requirements, they introduce two terms in the least-squares functional that are related to the conditions on the interface. They defined the least-squares functional including the integrations of jumps over the interface to impose the continuous interface conditions. Also one may find the least-squares finite element approaches for elliptic problems with discontinuous coefficients in [4] and [3]. The success of finite element least-squares method for such an interface problem stimulated the usage of pseudo-spectral methods or spectral element methods which is known as a very accurate method (see [2], [12], [16] and [24]). Furthermore, in [20] they analyzed the least-squares pseudo-spectral collocation method for elliptic problems without any interface. They also provided the analysis and numerical computations for Stokes equations in [21]. Therefore we believe that it is worth to develop the least-squares Legendre and Chebyshev pseudo-spectral methods to solve the first-order system corresponding to the interface problem (1.1). On the other hand, one may consider least-squares methods using negative norm or mesh dependent norm because the right hand side belongs to $H^{-1}(\Omega)$. Such approaches can be found in [6], [5] and [8] for the finite element approximation, and in [22] for the pseudo-spectral approximation. Instead of using negative norm, in [11] they developed the discrete FOSLS by directly approximating $H$ (div) $\cap H$ (curl)-type space based on the Helmholtz decomposition, in which under general assumptions they established error estimates in the $L^{2}$ - and $H^{1}$-norms for the vector and scalar variables, respectively. However, such methods need the global regularity for 
the solution so that the methods have a limited convergence with lack of global regularity.

In this paper, we introduce a pseudo-spectral least-squares method having full spectral convergence in an appropriate norm using two domain decomposition provided that the solution has local regularity enough in each sub-domain. To do this, we first apply a standard finite element argument to the problem (1.1) in order to derive two elliptic problems without any singular source term defined in each sub-domain, respectively. The singular source term resolved itself into a jump interface condition. See [23] and [28] for more details. Introducing the flux variable $\mathbf{u}=a \nabla p$, such two elliptic equations can be written as equivalent two first-order systems of linear equations which are very similar to the equations considered in [13]. Our interface condition for the trace of the normal components of the flux variable is discontinuous due to the singular source term. Such a jump condition is indeed given by $[\mathbf{n} \cdot \mathbf{u}]_{\Gamma}=\nu g(y)$. We define a solution space of functions satisfying not only essential boundary conditions but also jump interface conditions. That is, we include the jump interface conditions into the solution space as essential conditions. We then define two kinds of least-squares functionals summing the broken $L^{2}(\Omega)$-norms of residual equations of two systems. We do not add any integration over the interface to the least-squares functionals. The purpose of this paper is to show that the homogeneous least-squares functionals are equivalent to appropriate broken norms and the methods have spectral convergence in their norms. We also present the implementation for the proposed methods with some numerical results for two interface problems. In this paper, we analyze our methods for only Legendre spectral approximation. For the Chebyshev spectral approximation, we provide only numerical experiments, but one may easily analyze the method using the similar arguments of this paper together with some lemmas given in [20]. These approaches can be further applied to the finite element approximation.

This paper consists of as follows. In Section 2, we provide definitions, notations and basic known facts. In Sections 3, two kinds of least-squares pseudospectral collocation methods are presented including the norm equivalence and spectral convergence. In Section 4, we explain how the linear system can be set up and provide several numerical experiments including Legendre and Chebyshev approximations for interface problems.

\section{Preliminaries}

In this section, we provide some preliminaries, definitions and notations for future use. The standard notations and definitions are used for the weighted Sobolev spaces $H_{w}^{s}(\Omega)^{2}$ equipped with weighted inner products $(\cdot, \cdot)_{s, w}$ and corresponding weighted norms $\|\cdot\|_{s, w}, s \geq 0$, where $w(x, y)=\hat{w}(x) \hat{w}(y)$ is Legendre weight function when $\hat{w}(t)=1$ or Chebyshev weight function when $\hat{w}(t)=\frac{1}{\sqrt{1-t^{2}}}$. The space $H_{w}^{0}(\Omega)$ coincides with $L_{w}^{2}(\Omega)$, in which case the 
norm and inner product will be denoted by $\|\cdot\|_{w}$ and $(\cdot, \cdot)_{w}$, respectively. For Legendre case, we simply write the notations without the subscript $w$, for example, $H^{s}(\Omega):=H_{w}^{s}(\Omega),(\cdot, \cdot):=(\cdot, \cdot)_{w},\|\cdot\|:=\|\cdot\|_{w}$ if $w(x, y)=1$.

Let $\dot{H}_{w}^{s}(\Omega)$ be a subspace of $L_{w}^{2}(\Omega)$ given by

$$
\dot{H}_{w}^{s}(\Omega)=\left\{v=\left[v_{1}: v_{2}\right] \in L_{w}^{2}(\Omega)\left|v_{i}=v\right|_{\Omega_{i}} \in H_{w}^{s}\left(\Omega_{i}\right), i=1,2\right\}
$$

equipped with the so-called "broken" norm

$$
\|v\|_{\dot{H}_{w}^{s}(\Omega)}^{2}=\left\|\left.v\right|_{\Omega_{1}}\right\|_{H_{w}^{s}\left(\Omega_{1}\right)}^{2}+\left\|\left.v\right|_{\Omega_{2}}\right\|_{H_{w}^{s}\left(\Omega_{2}\right)}^{2} .
$$

Note that $\|v\|_{\dot{H}_{w}^{s}(\Omega)}^{2}=\|v\|_{H_{w}^{s}(\Omega)}^{2}$ if $v \in H_{w}^{s}(\Omega)$. Let $\mathcal{P}_{N}$ be the space of all polynomials of degree less than or equal to $N$. Let $\left\{\xi_{i}\right\}_{i=0}^{N}$ be the LGL or CGL points on $[-1,1]$ such that

$$
-1=: \xi_{0}<\xi_{1}<\cdots<\xi_{N-1}<\xi_{N}:=1 .
$$

For Legendre case, $\left\{\xi_{i}\right\}_{i=0}^{N}$ are the zeros of $\left(1-t^{2}\right) L_{N}^{\prime}(t)$ where $L_{N}$ is the $N^{t h}$ Legendre polynomial and the corresponding quadrature weights $\left\{w_{i}\right\}_{i=0}^{N}$ are given by

$$
\begin{aligned}
& w_{j}=\frac{2}{N(N+1)} \frac{1}{\left[L_{N}\left(\xi_{j}\right)\right]^{2}}, \quad 1 \leq j \leq N-1, \\
& w_{0}=w_{N}=\frac{2}{N(N+1)} .
\end{aligned}
$$

For Chebyshev case, $\left\{\xi_{i}\right\}_{i=0}^{N}$ are the zeros of $\left(1-t^{2}\right) T_{N}^{\prime}(t)$ where $T_{N}$ is the $N^{t h}$ Chebyshev polynomial and the corresponding quadrature weights $\left\{w_{i}\right\}_{i=0}^{N}$ are given by

$$
\begin{aligned}
& w_{j}=\frac{\pi}{N}, \quad 1 \leq j \leq N-1, \\
& w_{0}=w_{N}=\frac{\pi}{2 N}
\end{aligned}
$$

Then, we have the following LGL or CGL quadrature formula such that

$$
\int_{-1}^{1} p(t) \hat{w}(t) d t=\sum_{i=0}^{N} w_{i} p\left(\xi_{i}\right), \quad \forall p \in \mathcal{P}_{2 N-1} .
$$

Let $\left\{\phi_{i}\right\}_{i=0}^{N}$ be the set of Lagrange polynomials of degree $N$ with respect to LGL or CGL points $\left\{\xi_{i}\right\}_{i=0}^{N}$ which satisfy

$$
\phi_{i}\left(\xi_{j}\right)=\delta_{i j}, \quad \forall i, j=0,1, \ldots, N,
$$

where $\delta_{i j}$ denotes the Kronecker delta.

Denote by $\mathcal{Q}_{N}(D)$ the space of all polynomials, defined on $D$, of degree less than or equal to $N$ with respect to each single variable $x$ and $y$. Let

$$
\dot{\mathcal{Q}}_{N}=\left\{u \in L^{2}(\Omega):\left.u\right|_{\bar{\Omega}_{\ell}} \in \mathcal{Q}_{N}\left(\bar{\Omega}_{\ell}\right), \ell=1,2\right\} .
$$


Define the basis functions for $\dot{\mathcal{Q}}_{N}$ as

$$
\begin{aligned}
& \psi_{i j}^{1}(x, y)= \begin{cases}\phi_{i}(2 x+1) \phi_{j}(y) & \text { for }-1 \leq x \leq 0 \\
0 & \text { for } 0<x \leq 1,\end{cases} \\
& \psi_{i j}^{2}(x, y)= \begin{cases}0 & \text { for }-1 \leq x<0 \\
\phi_{i}(2 x-1) \phi_{j}(y) & \text { for } 0 \leq x \leq 1\end{cases}
\end{aligned}
$$

The two-dimensional LGL or CGL nodes $\left\{\mathrm{x}_{i j}^{\ell}\right\}$ and weights $\left\{\mathrm{w}_{i j}^{\ell}\right\}$ with respect to each domain $\Omega_{\ell}(\ell=1,2)$ are given by

$$
\mathrm{x}_{i j}^{\ell}=\left(\xi_{i}^{\ell}, \xi_{j}\right), \quad \mathrm{w}_{i j}=\frac{1}{2} w_{i} w_{j}, \quad i, j=0,1, \ldots, N, \ell=1,2,
$$

where

$$
\xi_{i}^{\ell}= \begin{cases}-1+\frac{1}{2}\left(1+\xi_{i}\right) & \text { for } \ell=1, \\ \frac{1}{2}\left(1+\xi_{i}\right) & \text { for } \ell=2 .\end{cases}
$$

For any piecewise continuous functions $u$ and $v$ on $\bar{\Omega}$, we define the discrete scalar product and norm respectively as

$$
\langle u, v\rangle_{w, N}=\sum_{\ell=1}^{2} \sum_{i, j=0}^{N} \mathrm{w}_{i j} u\left(\mathrm{x}_{i j}^{\ell}\right) v\left(\mathrm{x}_{i j}^{\ell}\right) \quad \text { and } \quad\|v\|_{w, N}=\langle v, v\rangle_{w, N}^{\frac{1}{2}} .
$$

Then, one may easily show from (2.1) that

$$
\langle u, v\rangle_{w, N}=(u, v)_{w}=\int_{\Omega} u v w d \mathrm{x} \quad \text { for } \quad u v \in \dot{\mathcal{Q}}_{2 N-1} .
$$

It is well-known that

$$
\|v\|_{w} \leq\|v\|_{w, N} \leq \gamma^{*}\|v\|_{w}, \quad \forall v \in \dot{\mathcal{Q}}_{N},
$$

where $\gamma^{*}=2+\frac{1}{N}$ for Legendre case and $\gamma^{*}=2$ for Chebyshev case.

For any piecewise continuous function $v$ on $\bar{\Omega}$, we define the interpolant $I_{N} v \in \dot{\mathcal{Q}}_{N}$ of $v$ at the LGL or CGL points as

$$
I_{N} v(\mathrm{x})=\sum_{\ell=1}^{2} \sum_{i, j=0}^{N} v\left(\mathrm{x}_{i j}^{\ell}\right) \psi_{i j}^{\ell}(\mathrm{x}), \quad \forall \mathrm{x} \in \bar{\Omega} .
$$

Using the results given in $[2,12,26]$, one may easily check the following interpolation error estimate given by

$$
\left\|v-I_{N} v\right\|_{\dot{H}_{w}^{k}(\Omega)} \leq C N^{k-s}\|v\|_{\dot{H}_{w}^{s}(\Omega)}, \quad k=0,1,
$$

provided $v \in \dot{H}_{w}^{s}(\Omega)$ for $s \geq 2$. Using (2.2)-(2.5) we can show that for any $u \in \dot{H}_{w}^{s}(\Omega), s \geq 2$, and any $v_{N} \in \dot{\mathcal{Q}}_{N}$

$$
\left|\left(u, v_{N}\right)_{w}-\left\langle u, v_{N}\right\rangle_{w, N}\right| \leq C N^{-s}\|u\|_{\dot{H}_{w}^{s}(\Omega)}\left\|v_{N}\right\|_{w}
$$

Throughout this paper, denotes a generic constant $C$ depending only on the physical domain $\Omega$ and coefficients given in the problem (1.1). 


\section{Pseudo-spectral least-squares method}

In this section, we investigate the Legendre pseudo-spectral least-squares method for the first-order system of linear equations equivalent to the problem (1.1). Throughout this section, we set $w(x, y)=1$.

For $\mathbf{v}=\left[\mathbf{v}_{1}: \mathbf{v}_{2}\right] \in\left[\dot{H}^{1}(\Omega)\right]^{2}$, along the interface $\Gamma$ denote by

$$
[\mathbf{n} \cdot \mathbf{v}]_{\Gamma}=\mathbf{n}_{1} \cdot \mathbf{v}_{1}+\mathbf{n}_{2} \cdot \mathbf{v}_{2} \quad \text { and } \quad\left[\boldsymbol{\tau} \cdot a^{-1} \mathbf{v}\right]_{\Gamma}=a_{1}^{-1} \boldsymbol{\tau}_{1} \cdot \mathbf{v}_{1}+a_{2}^{-1} \boldsymbol{\tau}_{2} \cdot \mathbf{v}_{2},
$$

where $a_{i}:=\left.a\right|_{\Omega_{i}}$ denotes the constant diffusion coefficient over subdomain $\Omega_{i}$, and $\mathbf{n}_{i}$ and $\boldsymbol{\tau}_{i}$ are unit normal and tangent vectors on $\Omega_{i}$, respectively. Let $\nabla \times$ denote the curl operator given by $\nabla \times \mathbf{v}=\partial_{x} v_{2}-\partial_{y} v_{1}$ for a vector function $\mathbf{v}=\left(v_{1}, v_{2}\right)^{T}$.

In order to solve the problem (1.1) via the interface problem, we need to first derive the jump conditions from the singular source term $s(x, y)=\nu \delta(x) g(y)$. By a standard finite element argument, we can derive the following jump condition (see [23] and [28]), for $p=\left[p_{1}: p_{2}\right] \in H^{1}(\Omega)$

$$
[\mathbf{n} \cdot(a \nabla p)]_{\Gamma}=a_{1} \partial_{x} p_{1}(0, y)-a_{2} \partial_{x} p_{2}(0, y)=\nu g(y) \text { for all } y \in(-1,1) .
$$

\subsection{Least-squares of $\left[\dot{H}^{1}(\Omega)^{2} \times H^{1}(\Omega)\right]$-norm equivalence}

In this subsection, we establish the pseudo-spectral least-squares method whose homogeneous functional is equivalent to $\left[\dot{H}^{1}(\Omega)^{2} \times H^{1}(\Omega)\right]$-norm.

For a simple analysis, we consider the function $g(y)$ as its LGL-interpolant $I_{N} g$, i.e., $g=I_{N} g$. Let

$$
V:=\left\{q \in H^{1}(\Omega): q=0 \text { on } \Gamma_{D}\right\},
$$

and let $\mathbf{W}$ be a space of vector functions:

$$
\begin{gathered}
\mathbf{W}:=\left\{\mathbf{v} \in\left[L^{2}(\Omega)\right]^{2}: \nabla \cdot \mathbf{v} \in \dot{L}^{2}(\Omega), \nabla \times\left(a^{-1} \mathbf{v}\right) \in L^{2}(\Omega), \mathbf{n} \cdot \mathbf{v}=0 \text { on } \Gamma_{N},\right. \\
\left.\boldsymbol{\tau} \cdot a^{-1} \mathbf{v}=0 \text { on } \Gamma_{D},[\mathbf{n} \cdot \mathbf{v}]_{\Gamma}=\nu g(y) \text { and }\left[\boldsymbol{\tau} \cdot a^{-1} \mathbf{v}\right]_{\Gamma}=0\right\}
\end{gathered}
$$

equipped with the norm

$$
\|\mathbf{v}\|_{\mathbf{W}}^{2}=\|\mathbf{v}\|_{L^{2}}^{2}+\|\nabla \cdot \mathbf{v}\|_{\dot{L}^{2}(\Omega)}^{2}+\left\|\nabla \times\left(a^{-1} \mathbf{v}\right)\right\|_{L^{2}(\Omega)}^{2} .
$$

If $\nu=0$, then $\mathbf{W} \subset H(\operatorname{div} ; \Omega) \cap H\left(\operatorname{curl} a^{-1} ; \Omega\right)$ where

$$
H(\operatorname{div} ; \Omega)=\left\{\mathbf{v} \in\left[L^{2}(\Omega)\right]^{2}: \nabla \cdot \mathbf{v} \in L^{2}(\Omega)\right\}
$$

equipped with the norm

$$
\|\mathbf{v}\|_{H(\operatorname{div} ; \Omega)}^{2}=\|\mathbf{v}\|_{L^{2}(\Omega)}^{2}+\|\nabla \cdot \mathbf{v}\|_{L^{2}(\Omega)}^{2}
$$

and

$$
H\left(\operatorname{curl} a^{-1} ; \Omega\right)=\left\{\mathbf{v} \in\left[L^{2}(\Omega)\right]^{2}: \nabla \times\left(a^{-1} \mathbf{v}\right) \in L^{2}(\Omega)\right\}
$$

equipped with the norm

$$
\|\mathbf{v}\|_{H\left(\operatorname{curl} a^{-1} ; \Omega\right)}^{2}=\|\mathbf{v}\|_{L^{2}(\Omega)}^{2}+\left\|\nabla \times\left(a^{-1} \mathbf{v}\right)\right\|_{L^{2}(\Omega)}^{2} .
$$


One may show from [10] that there exists a constant $C>0$ such that

$$
\frac{1}{C}\|\mathbf{v}\|_{\dot{H}^{1}(\Omega)}^{2} \leq\|\mathbf{v}\|_{\mathbf{W}}^{2} \leq C\|\mathbf{v}\|_{\dot{H}^{1}(\Omega)}^{2}
$$

so that $\mathbf{W}$ is a subspace of $\left[\dot{H}^{1}(\Omega)\right]^{2}$. Let

$$
\mathbf{W}_{N}=\mathbf{W} \cap\left[\dot{\mathcal{Q}}_{N}\right]^{2} \text { and } \quad V_{N}=V \cap \dot{\mathcal{Q}}_{N} .
$$

Introducing the flux variable $\mathbf{u}=a \nabla p$ and using the identities

$$
\nabla \times a^{-1} \mathbf{u}=0 \quad \text { in } \Omega \text { and } \boldsymbol{\tau} \cdot a^{-1} \mathbf{u}=0 \text { on } \Gamma_{D},
$$

we have the first-order system of linear equations equivalent to (1.1) such that

$$
\left\{\begin{array}{rlll}
\mathbf{u}-a \nabla p & =\mathbf{0} & & \text { in } \Omega, \\
-\nabla \cdot \mathbf{u}+\mathbf{b} \cdot \nabla p+c_{0} p & = & & \text { in } \Omega, \\
\nabla \times\left(a^{-1} \mathbf{u}\right) & = & & \text { in } \Omega, \\
p & = & & \text { on } \Gamma_{D}, \\
\mathbf{n} \cdot \mathbf{u} & = & & \text { on } \Gamma_{N}, \\
\boldsymbol{\tau} \cdot\left(a^{-1} \mathbf{u}\right) & = & & \text { on } \Gamma_{D} .
\end{array}\right.
$$

Define the least-squares functional for the system (3.1) as

$G(\mathbf{v}, q ; f)=\left\|f+\nabla \cdot \mathbf{v}-\mathbf{b} \cdot \nabla q-c_{0} q\right\|_{\dot{L}^{2}(\Omega)}^{2}+\|\mathbf{v}-a \nabla q\|_{\dot{L}^{2}(\Omega)}^{2}+\left\|\nabla \times\left(a^{-1} \mathbf{v}\right)\right\|_{\dot{L}^{2}(\Omega)}^{2}$ for $(\mathbf{v}, q) \in \mathbf{W} \times V$. The first-order system least-squares variational problem for (3.1) is to minimize the quadratic functional $G(\mathbf{v}, q ; f)$ over $\mathbf{W} \times V$ : find $(\mathbf{u}, p) \in \mathbf{W} \times V$ such that

$$
G(\mathbf{u}, p ; f)=\inf _{(\mathbf{v}, q) \in \mathbf{W} \times V} G(\mathbf{v}, q ; f) .
$$

The corresponding variational problem is to find $(\mathbf{u}, p) \in \mathbf{W} \times V$ such that

$$
b(\mathbf{u}, p ; \mathbf{v}, q)=f(\mathbf{v}, q) \quad \forall(\mathbf{v}, q) \in \mathbf{W} \times V,
$$

where the bilinear form $a(\cdot ; \cdot)$ is given by

$$
\begin{aligned}
b(\mathbf{u}, p ; \mathbf{v}, q)= & \left(\nabla \cdot \mathbf{u}-\mathbf{b} \cdot \nabla p-c_{0} p, \nabla \cdot \mathbf{v}-\mathbf{b} \cdot \nabla q-c_{0} q\right)_{\dot{L}^{2}(\Omega)} \\
& +(\mathbf{u}-a \nabla p, \mathbf{v}-a \nabla q)_{\dot{L}^{2}(\Omega)}+\left(\nabla \times\left(a^{-1} \mathbf{u}\right), \nabla \times\left(a^{-1} \mathbf{v}\right)\right)_{\dot{L}^{2}(\Omega)}
\end{aligned}
$$

and the linear form $f(\cdot)$ is given by

$$
f(\mathbf{v}, q)=-\left(f, \nabla \cdot \mathbf{v}-\mathbf{b} \cdot \nabla q-c_{0} q\right)_{\dot{L}^{2}(\Omega)} .
$$

Define

$$
\begin{aligned}
G_{\ell}(\mathbf{v}, q ; f)= & \left\|f+\nabla \cdot \mathbf{v}-\mathbf{b} \cdot \nabla q-c_{0} q\right\|_{L^{2}\left(\Omega_{\ell}\right)}^{2} \\
& +\|\mathbf{v}-a \nabla q\|_{L^{2}\left(\Omega_{\ell}\right)}^{2}+\left\|\nabla \times\left(a^{-1} \mathbf{v}\right)\right\|_{L^{2}\left(\Omega_{\ell}\right)}^{2} \quad \text { for } \ell=1,2 .
\end{aligned}
$$

Then we have

$$
G(\mathbf{v}, q ; f)=G_{1}(\mathbf{v}, q ; f)+G_{2}(\mathbf{v}, q ; f) .
$$

In this paper, we assume that there exists a unique solution $(p, \mathbf{u}) \in \dot{H}^{k}(\Omega) \times$ $\left[\dot{H}^{k-1}(\Omega)\right]^{2}$ with $k \geq 2$ for problem (3.1). See [27] for more details. 
The following theorem establishes the continuity and ellipticity of the bilinear form $a(\cdot ; \cdot)$ so that there exists a unique solution for the problem (3.4).

Theorem 3.1. For any $(\mathbf{v}, q) \in \mathbf{W} \times V$, there exists a positive constant $C$ such that

$$
\frac{1}{C}\left(\|\mathbf{v}\|_{\dot{H}^{1}(\Omega)}^{2}+\|q\|_{H^{1}(\Omega)}^{2}\right) \leq G(\mathbf{v}, q ; 0) \leq C\left(\|\mathbf{v}\|_{\dot{H}^{1}(\Omega)}^{2}+\|q\|_{H^{1}(\Omega)}^{2}\right) .
$$

Proof. The functional $G_{\ell}(\cdot, \cdot ; \cdot), i=1,2$, is a special case of the general form given in [10]. Using the similar argument given in [10], we can easily show that there exists a constant $C>0$ such that, for $i=1,2$,

$$
\frac{1}{C}\left(\|\mathbf{v}\|_{H^{1}\left(\Omega_{\ell}\right)}^{2}+\|q\|_{H^{1}\left(\Omega_{\ell}\right)}^{2}\right) \leq G_{\ell}(\mathbf{v}, q ; 0) \leq C\left(\|\mathbf{v}\|_{H^{1}\left(\Omega_{\ell}\right)}^{2}+\|q\|_{H^{1}\left(\Omega_{\ell}\right)}^{2}\right) .
$$

This completes the proof of the theorem.

Define the discrete least-squares functional using the discrete spectral norm as

(3.5) $G_{N}(\mathbf{v}, q ; f)=\left\|f+\nabla \cdot \mathbf{v}-\mathbf{b} \cdot \nabla q-c_{0} q\right\|_{N}^{2}+\|\mathbf{v}-a \nabla q\|_{N}^{2}+\left\|\nabla \times\left(a^{-1} \mathbf{v}\right)\right\|_{N}^{2}$

for $(\mathbf{v}, q) \in \mathbf{W}_{N} \times V_{N}$. The discrete least-squares problem associated to (3.5) is then to minimize the quadratic functional $G_{N}(\mathbf{v}, q ; f)$ over $\mathbf{W}_{N} \times V_{N}$ and the corresponding variational problem (Legendre pseudo-spectral collocation problem) is to find $\left(\mathbf{u}_{N}, p_{N}\right) \in \mathbf{W}_{N} \times V_{N}$ such that

$$
b_{N}\left(\mathbf{u}_{N}, p_{N} ; \mathbf{v}, q\right)=f_{N}(\mathbf{v}, q), \quad \forall(\mathbf{v}, q) \in \mathbf{W}_{N} \times V_{N},
$$

where the discrete bilinear form $a_{N}(\cdot ; \cdot)$ and linear form $f_{N}(\cdot)$ are given by

$$
\begin{aligned}
b_{N}\left(\mathbf{u}_{N}, p_{N} ; \mathbf{v}, q\right)= & \left\langle\nabla \cdot \mathbf{u}_{N}-\mathbf{b} \cdot \nabla p_{N}-c_{0} p_{N}, \nabla \cdot \mathbf{v}-\mathbf{b} \cdot \nabla q-c_{0} q\right\rangle_{N} \\
& +\left\langle\mathbf{u}_{N}-a \nabla p_{N}, \mathbf{v}-a \nabla q\right\rangle_{N} \\
& +\left\langle\nabla \times\left(a^{-1} \mathbf{u}_{N}\right), \nabla \times\left(a^{-1} \mathbf{v}\right)\right\rangle_{N}
\end{aligned}
$$

and

$$
f_{N}(\mathbf{v}, q)=-\left\langle f, \nabla \cdot \mathbf{v}-\mathbf{b} \cdot \nabla q-c_{0} q\right\rangle_{N} .
$$

In [20], they established the least-squares pseudo-spectral collocation method for an elliptic equations with continuous coefficients. The similar arguments given in [20] yields the following theorem which shows the continuity and ellipticity of the discrete functional $G_{N}(\cdot ; 0)$.

Theorem 3.2. For any $(\mathbf{v}, q) \in \mathbf{W}_{N} \times V_{N}$, there exists a constant $C$ such that

$$
\frac{1}{C}\left(\|\mathbf{v}\|_{\dot{H}^{1}(\Omega)}^{2}+\|q\|_{H^{1}(\Omega)}^{2}\right) \leq G_{N}(\mathbf{v}, q ; 0) \leq C\left(\|\mathbf{v}\|_{\dot{H}^{1}(\Omega)}^{2}+\|q\|_{H^{1}(\Omega)}^{2}\right) .
$$

Using the same technique for a generalized Galerkin method given in [2] and [26], one may easily obtain the spectral convergence in $\left[\dot{H}^{1}(\Omega)\right]^{2} \times H^{1}(\Omega)$ for the proposed method in the following theorem. The similar results can be found in [20] and [21]. 
Theorem 3.3. Assume that the solution $(\mathbf{u}, p)$ of $(3.1)$ is in $\left(\left[\dot{H}^{s}(\Omega)\right]^{2} \cap \mathbf{W}\right) \times$ $\left(\dot{H}^{s}(\Omega) \cap V\right)$ for some $s \geq 1$ and $f \in \dot{H}^{\ell}(\Omega)$ for some integer $\ell \geq 2$. Let $\left(\mathbf{u}_{N}, p_{N}\right) \in \mathbf{W}_{N} \times V_{N}$ be the discrete solution of the problem (3.6). Then there exists a constant $C$ such that

$$
\begin{aligned}
& \left\|\mathbf{u}-\mathbf{u}_{N}\right\|_{\dot{H}^{1}(\Omega)}+\left\|p-p_{N}\right\|_{H^{1}(\Omega)} \\
\leq & C\left[N^{1-s}\left(\|\mathbf{u}\|_{\dot{H}^{s}(\Omega)}+\|p\|_{\dot{H}^{s}(\Omega)}\right)+N^{-\ell}\|f\|_{\dot{H}^{\ell}(\Omega)}\right] .
\end{aligned}
$$

\subsection{Least-squares of $\left[H(\operatorname{div} ; \Omega) \times H^{1}(\Omega)\right]$-norm equivalence}

In this subsection, we establish the pseudo-spectral least-squares method whose homogeneous functional is equivalent to $\left[H(\operatorname{div} ; \Omega) \times H^{1}(\Omega)\right]$-norm.

Let $\mathbf{W}^{\text {div }}$ be a subspace of $H(\operatorname{div} ; \Omega)$ for vector functions:

$$
\mathbf{W}^{\operatorname{div}}:=\left\{\mathbf{v} \in\left[L^{2}(\Omega)\right]^{2}: \nabla \cdot \mathbf{v} \in \dot{L}^{2}(\Omega), \mathbf{n} \cdot \mathbf{v}=0 \text { on } \Gamma_{N},[\mathbf{n} \cdot \mathbf{v}]_{\Gamma}=\nu g(y)\right\}
$$

equipped with the norm

$$
\|\mathbf{v}\|_{\mathbf{W}^{\text {div }}}^{2}=\|\mathbf{v}\|_{L^{2}(\Omega)}^{2}+\|\nabla \cdot \mathbf{v}\|_{\dot{L}^{2}(\Omega)}^{2} .
$$

Note that $\mathbf{W}^{\operatorname{div}} \subsetneq H(\operatorname{div} ; \Omega)$ and $\|\mathbf{v}\|_{\mathbf{W}^{\text {div }}}=\|\mathbf{v}\|_{H(\operatorname{div} ; \Omega)}$ if $\nu=0$. Let

$$
\mathbf{W}_{N}^{\text {div }}=\mathbf{W}^{\text {div }} \cap\left[\dot{\mathcal{Q}}_{N}\right]^{2} .
$$

Introducing the flux variable $\mathbf{u}=a \nabla p$, we have the first-order system of linear equations equivalent to (1.1) such that

$$
\left\{\begin{array}{rlll}
\mathbf{u}-a \nabla p & =\mathbf{0} & & \text { in } \Omega, \\
-\nabla \cdot \mathbf{u}+\mathbf{b} \cdot \nabla p+c_{0} p & = & & \text { in } \Omega, \\
p & = & 0 & \text { on } \Gamma_{D} \\
\mathbf{n} \cdot \mathbf{u} & = & & \text { on } \Gamma_{N} .
\end{array}\right.
$$

Define the least-squares functional for the system (3.7) as

$$
G^{\operatorname{div}}(\mathbf{v}, q ; f)=\left\|f+\nabla \cdot \mathbf{v}-\mathbf{b} \cdot \nabla q-c_{0} q\right\|_{\dot{L}^{2}(\Omega)}^{2}+\|\mathbf{v}-a \nabla q\|_{\dot{L}^{2}(\Omega)}^{2}
$$

for $(\mathbf{v}, q) \in \mathbf{W}^{\text {div }} \times V$. The least-squares problem associated to (3.8) is then to minimize the quadratic functional $G^{\operatorname{div}}(\mathbf{v}, q ; f)$ over $\mathbf{W}^{\text {div }} \times V$, and the corresponding variational problem is to find $(\mathbf{u}, p) \in \mathbf{W}^{\text {div }} \times V$ such that

$$
b(\mathbf{u}, p ; \mathbf{v}, q)=f(\mathbf{v}, q) \quad \forall(\mathbf{v}, q) \in \mathbf{W}^{\operatorname{div}} \times V,
$$

where the bilinear form $b(\cdot ; \cdot)$ is given by

$$
\begin{aligned}
b(\mathbf{u}, p ; \mathbf{v}, q)= & \left(\nabla \cdot \mathbf{u}-\mathbf{b} \cdot \nabla p-c_{0} p, \nabla \cdot \mathbf{v}-\mathbf{b} \cdot \nabla q-c_{0} q\right)_{\dot{L}^{2}(\Omega)} \\
& +(\mathbf{u}-a \nabla p, \mathbf{v}-a \nabla q)_{\dot{L}^{2}(\Omega)}
\end{aligned}
$$

and the linear form $f(\cdot)$ is given by

$$
f(\mathbf{v}, q)=-\left(f, \nabla \cdot \mathbf{v}-\mathbf{b} \cdot \nabla q-c_{0} q\right)_{\dot{L}^{2}(\Omega)} .
$$

Define, for $\ell=1,2$,

$$
G_{\ell}^{\operatorname{div}}(\mathbf{v}, q ; f)=\left\|f+\nabla \cdot \mathbf{v}-\mathbf{b} \cdot \nabla q-c_{0} q\right\|_{L^{2}\left(\Omega_{\ell}\right)}^{2}+\|\mathbf{v}-a \nabla q\|_{L^{2}\left(\Omega_{\ell}\right)}^{2}
$$


so that

$$
G^{\operatorname{div}}(\mathbf{v}, q ; f)=G_{1}^{\operatorname{div}}(\mathbf{v}, q ; f)+G_{2}^{\operatorname{div}}(\mathbf{v}, q ; f) .
$$

The following theorem establishes the continuity and ellipticity of the bilinear form $b(\cdot ; \cdot)$ so that there exists a unique solution for the problem (3.9).

Theorem 3.4. For any $(\mathbf{v}, q) \in \mathbf{W}^{\text {div }} \times V$, there exists a positive constant $C$ such that

$$
\frac{1}{C}\left(\|\mathbf{v}\|_{\mathbf{W}^{d i v}}^{2}+\|q\|_{H^{1}(\Omega)}^{2}\right) \leq G^{d i v}(\mathbf{v}, q ; 0) \leq C\left(\|\mathbf{v}\|_{\mathbf{W}^{d i v}}^{2}+\|q\|_{H^{1}(\Omega)}^{2}\right) .
$$

Proof. The functional $G_{\ell}^{\operatorname{div}}(\cdot, \cdot ; \cdot), i=1,2$, is a special case of the general form given in [9]. Using the similar argument given in [10] and Theorem 3.1, one may easily show the conclusion.

Define the discrete least-squares functional using the discrete spectral norm as

$$
G_{N}^{\mathrm{div}}(\mathbf{v}, q ; f)=\left\|f+\nabla \cdot \mathbf{v}-\mathbf{b} \cdot \nabla q-c_{0} q\right\|_{N}^{2}+\|\mathbf{v}-a \nabla q\|_{N}^{2}
$$

for $(\mathbf{v}, q) \in \mathbf{W}_{N}^{\text {div }} \times V_{N}$. The discrete least-squares problem associated to (3.10) is then to minimize the quadratic functional $G_{N}^{\mathrm{div}}(\mathbf{v}, q ; f)$ over $\mathbf{W}_{N}^{\mathrm{div}} \times V_{N}$ and the corresponding variational problem is to find $\left(\mathbf{u}_{N}, p_{N}\right) \in \mathbf{W}_{N}^{\text {div }} \times V_{N}$ such that

$$
b_{N}\left(\mathbf{u}_{N}, p_{N} ; \mathbf{v}, q\right)=f_{N}(\mathbf{v}, q), \quad \forall(\mathbf{v}, q) \in \mathbf{W}_{N}^{\text {div }} \times V_{N},
$$

where the discrete bilinear form $b_{N}(\cdot ; \cdot)$ and linear form $f_{N}(\cdot)$ are given by

$$
\begin{aligned}
b_{N}\left(\mathbf{u}_{N}, p_{N} ; \mathbf{v}, q\right)= & \left\langle\nabla \cdot \mathbf{u}_{N}-\mathbf{b} \cdot \nabla p_{N}-c_{0} p_{N}, \nabla \cdot \mathbf{v}-\mathbf{b} \cdot \nabla q-c_{0} q\right\rangle_{N} \\
& +\left\langle\mathbf{u}_{N}-a \nabla p_{N}, \mathbf{v}-a \nabla q\right\rangle_{N}
\end{aligned}
$$

and

$$
f_{N}(\mathbf{v}, q)=-\left\langle f, \nabla \cdot \mathbf{v}-\mathbf{b} \cdot \nabla q-c_{0} q\right\rangle_{N}
$$

Using similar arguments given in [20], one may easily show the following theorem which shows the continuity and ellipticity of the discrete functional $G_{N}^{\operatorname{div}}(\cdot ; 0)$.

Theorem 3.5. For any $(\mathbf{v}, q) \in \mathbf{W}_{N} \times V_{N}$, there exists a constant $C$ such that

$$
\frac{1}{C}\left(\|\mathbf{v}\|_{\mathbf{W}^{d i v}}^{2}+\|q\|_{H^{1}(\Omega)}^{2}\right) \leq G_{N}(\mathbf{v}, q ; 0) \leq C\left(\|\mathbf{v}\|_{\mathbf{W}^{d i v}}^{2}+\|q\|_{H^{1}(\Omega)}^{2}\right) .
$$

The proposed method appears indeed as a generalized Galerkin method. We have the following convergence result using the same techniques given in [2] and $[26]$.

Theorem 3.6. Assume that the solution $(\mathbf{u}, p)$ of $(3.7)$ is in $\left(\left[\dot{H}^{s}(\Omega)\right]^{2} \cap\right.$ $\left.\mathbf{W}^{\text {div }}\right) \times\left(\dot{H}^{s}(\Omega) \cap V\right)$ for some $s \geq 1$ and $f \in \dot{H}^{\ell}(\Omega)$ for some integer $\ell \geq 2$. 
Let $\left(\mathbf{u}_{N}, p_{N}\right) \in \mathbf{W}_{N}^{\text {div }} \times V_{N}$ be the discrete solution of the problem (3.11). Then there exists a constant $C$ such that

$$
\begin{aligned}
& \left\|\mathbf{u}-\mathbf{u}_{N}\right\|_{\mathbf{W}^{d i v}}+\left\|p-p_{N}\right\|_{H^{1}(\Omega)} \\
\leq & C\left[N^{1-s}\left(\|\mathbf{u}\|_{\dot{H}^{s}(\Omega)}+\|p\|_{\dot{H}^{s}(\Omega)}\right)+N^{-\ell}\|f\|_{\dot{H}^{\ell}(\Omega)}\right] .
\end{aligned}
$$

Remark 3.7 (Chebyshev spectral collocation method). In this section, we have investigated the least-squares Legendre spectral collocation method for an elliptic equation with an interface. In [20] and [21], they provided the least-squares Legendre and Chebyshev spectral collocation method for elliptic equations without any interface. One may easily explore the Chebyshev spectral least-squares method using the similar arguments given in [20]. Lemma 4.1 through Lemma 4.3 of [20] can play important roles in the analysis of the Chebyshev pseudo-spectral approximation for the interface problem (1.1).

\section{Implementation and numerical results}

\subsection{Implementation}

The computation for the problems (3.6) and (3.11) can be easily implemented by using one-dimensional pseudo-spectral matrix and tensor product for both Legendre and Chebyshev pseudo-spectral approximations. See [20] for more details. Here, we briefly introduce an easy way to control the interface conditions imposed in the solution spaces $\mathbf{W}_{N}$ and $\mathbf{W}_{N}^{\text {div }}$. A similar technique can be found in [28].

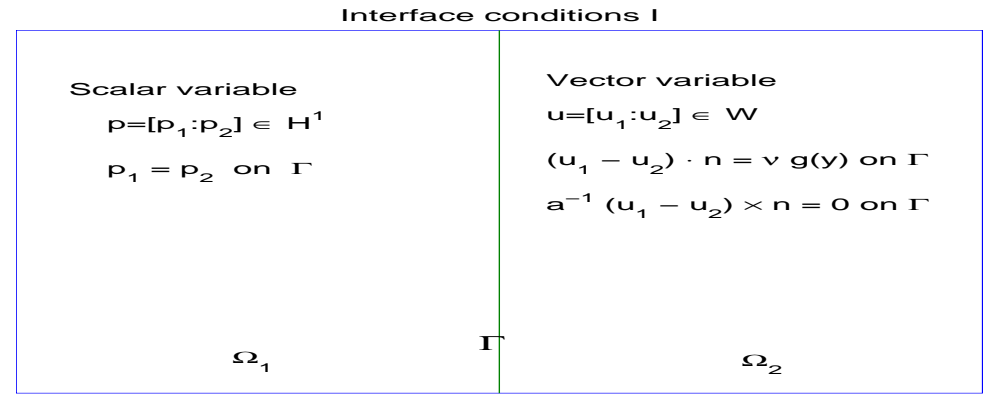

FiguRE 1. Decomposition of domain $\Omega$ and interface conditions when $\nu=0$.

In the solution space $\mathbf{W}_{N}$, we have the following interface conditions:

$$
[\mathbf{n} \cdot \mathbf{u}]_{\Gamma}=\nu g(y) \text { and }\left[\boldsymbol{\tau} \cdot a^{-1} \mathbf{u}\right]_{\Gamma}=0,
$$

where $\mathbf{u}=\left[\mathbf{u}^{1}: \mathbf{u}^{2}\right]$ with $\mathbf{u}^{i}=\left.\mathbf{u}\right|_{\Omega_{i}}$ and $\mathbf{u}^{i}=\left(u_{1}^{i}, u_{2}^{i}\right)^{T}$ for each $i=1,2$. Since $\left.\mathbf{n}\right|_{\Omega_{1}}=(1,0)$ and $\left.\boldsymbol{\tau}\right|_{\Omega_{1}}=(0,1)$, one may see the following jump conditions for both components:

$$
u_{1}^{2}=u_{1}^{1}-\nu g(y) \quad \text { and } \quad u_{2}^{2}=\left(a_{2} / a_{1}\right) u_{2}^{1} \quad \text { on } \quad \Gamma,
$$

where $a_{i}=\left.a\right|_{\Omega_{i}}$ for each $i=1,2$. Then, we can consider the discontinuous high-order approximation with interface conditions. 
We introduce an easy technique to impose the jump condition into the algebraic system for the case of only one variable problem. Consider the following problem:

$$
\text { Find } u=\left[u^{1}: u^{2}\right] \in W_{N} \text { such that } d(u, v)=(f, v) \quad \forall v \in W_{N}
$$

with a known jump condition:

$$
u^{2}=\alpha u^{1}-h(y) \quad \text { on } \quad \Gamma
$$

where $W_{N}$ is a finite dimensional space and $d(\cdot, \cdot)$ is a bilinear form.

Suppose that the resulting algebraic system for the problem (4.1) based on the discontinuous high-order approximation is

$$
\mathbf{A} \mathbf{U}:=\left[\begin{array}{lllll}
A_{11} & A_{12} & : & A_{13} & A_{14} \\
A_{21} & A_{22} & : & A_{23} & A_{24} \\
\cdots & \cdots & : & \cdots & \cdots \\
A_{31} & A_{32} & : & A_{33} & A_{34} \\
A_{41} & A_{42} & : & A_{43} & A_{44}
\end{array}\right]\left[\begin{array}{c}
\hat{u}_{I}^{1} \\
\hat{u}_{\Gamma}^{1} \\
\cdots \\
\hat{u}_{\Gamma}^{2} \\
\hat{u}_{I}^{2}
\end{array}\right]=\left[\begin{array}{c}
\hat{f}_{I}^{1} \\
\hat{f}_{\Gamma}^{1} \\
\cdots \\
\hat{f}_{\Gamma}^{2} \\
\hat{f}_{I}^{2}
\end{array}\right]
$$

where, for each $\ell=1,2, \hat{u}_{I}^{\ell}$ denotes the vector containing all nodal values of $u^{\ell}$ except nodal values on $\Gamma$ and $\hat{u}_{\Gamma}^{\ell}$ the vector containing the nodal values of $u^{\ell}$ only on $\Gamma$. Let $\hat{h}_{\Gamma}$ be the vector containing the nodal values of the function $h(y)$. From the jump condition (4.2), we can replace $\hat{u}_{\Gamma}^{2}$ by $\alpha \hat{u}_{\Gamma}^{1}-\hat{h}_{\Gamma}$ so that we obtain the following reduced algebraic system:

$$
\widehat{\mathbf{A}} \widehat{\mathbf{U}}:=\left[\begin{array}{lll}
A_{11} & A_{12}+\alpha A_{13} & A_{14} \\
A_{21} & A_{22}+\alpha A_{23} & A_{24} \\
A_{41} & A_{42}+\alpha A_{43} & A_{44}
\end{array}\right]\left[\begin{array}{l}
\hat{u}_{I}^{1} \\
\hat{u}_{\Gamma}^{1} \\
\hat{u}_{I}^{2}
\end{array}\right]=\left[\begin{array}{l}
\hat{f}_{I}^{1}+A_{13} \hat{h}_{\Gamma} \\
\hat{f}_{\Gamma}^{1}+A_{23} \hat{h}_{\Gamma} \\
\hat{f}_{I}^{2}+A_{43} \hat{h}_{\Gamma}
\end{array}\right] .
$$

In this case, we cannot guarantee the symmetry of $\widehat{\mathbf{A}}$ even though $\mathbf{A}$ is symmetric. To obtain a symmetric system, we add $\alpha$ times the third row-block to the second row-block so that the matrix $\mathbf{A}_{S}$ is symmetric if $\mathbf{A}$ is symmetric:

$$
\mathbf{A}_{S} \widehat{\mathbf{U}}=\widehat{\mathbf{F}}
$$

$$
\mathbf{A}_{S}=\left[\begin{array}{ccc}
A_{11} & A_{12}+\alpha A_{13} & A_{14} \\
& & \\
A_{21}+\alpha A_{31} & A_{22}+\alpha A_{23} & A_{24}+\alpha A_{34} \\
& +A_{32}+\alpha A_{33} & \\
A_{41} & A_{42}+\alpha A_{43} & A_{44}
\end{array}\right], \widehat{\mathbf{F}}=\left[\begin{array}{c}
\hat{f}_{I}^{1}+A_{13} \hat{h}_{\Gamma} \\
\hat{f}_{\Gamma}^{1}+A_{23} \hat{h}_{\Gamma} \\
+\alpha\left(\hat{f}_{\Gamma}^{2}+A_{33} \hat{h}_{\Gamma}\right) \\
\hat{f}_{I}^{2}+A_{43} \hat{h}_{\Gamma}
\end{array}\right] .
$$

After we compute $\hat{u}_{\Gamma}^{1}$, we set $\hat{u}_{\Gamma}^{2}=\alpha \hat{u}_{\Gamma}^{1}-\hat{h}_{\Gamma}$. On the other hand, the conforming approximation is nothing but only the case of $\alpha=1$ and $h(y)=0$. 


\subsection{Numerical results}

In this section, we present the numerical experiments for the first-order system (3.1) associated to the following elliptic partial differential equation (1.1).

Example 1 (Discontinuous diffusion coefficient). Consider the following problem without the singular source term:

$$
\left\{\begin{aligned}
-\nabla \cdot(a \nabla p)+\mathbf{b} \cdot \nabla p+c_{0} p & =f \quad \text { in } \Omega \\
p & =0 \quad \text { on } \partial \Omega .
\end{aligned}\right.
$$

The discontinuous diffusion coefficient $a(x, y)$ is given by

$$
a(x, y)= \begin{cases}1, & x \leq 0 \\ \sigma, & x>0\end{cases}
$$

In this example, we present some numerical results with the following exact solution

$$
p(x, y)=\left\{\begin{array}{cc}
\left((\sigma-2)(x+1)^{2}+(4-\sigma)(x+1)\right) \sin \left(\frac{\pi}{2}(y+1)\right), & x \leq 0 \\
\left(-3(x+1)^{2}+7(x+1)-2\right) \sin \left(\frac{\pi}{2}(y+1)\right), & x>0
\end{array}\right.
$$

The right hand side $f$ is given by the direct computation of the left hand side with the solution $p$. One may easily see that $p \in H^{2}(\Omega)$ and $\mathbf{u}=a(x, y) \nabla p \in$ $\left[H^{1}(\Omega)\right]^{2}$, but $\mathbf{u} \notin\left[H^{2}(\Omega)\right]^{2}$ when $\sigma=1$. However, if $\sigma \neq 1$, then $p \in H^{1}(\Omega)$ and $\mathbf{u} \in \mathbf{W} \subset \mathbf{W}^{\text {div }}$, but $\mathbf{u} \notin\left[H^{1}(\Omega)\right]^{2}$. The numerical tests will be performed with $\sigma=1$ and 100. Let $e_{\mathbf{u}}=\mathbf{u}-\mathbf{u}_{N}$ and $e_{p}=p-p_{N}$ be the discretization errors where $\left(\mathbf{u}_{N}, p_{N}\right)$ is the approximate solution to (3.6) or (3.11).
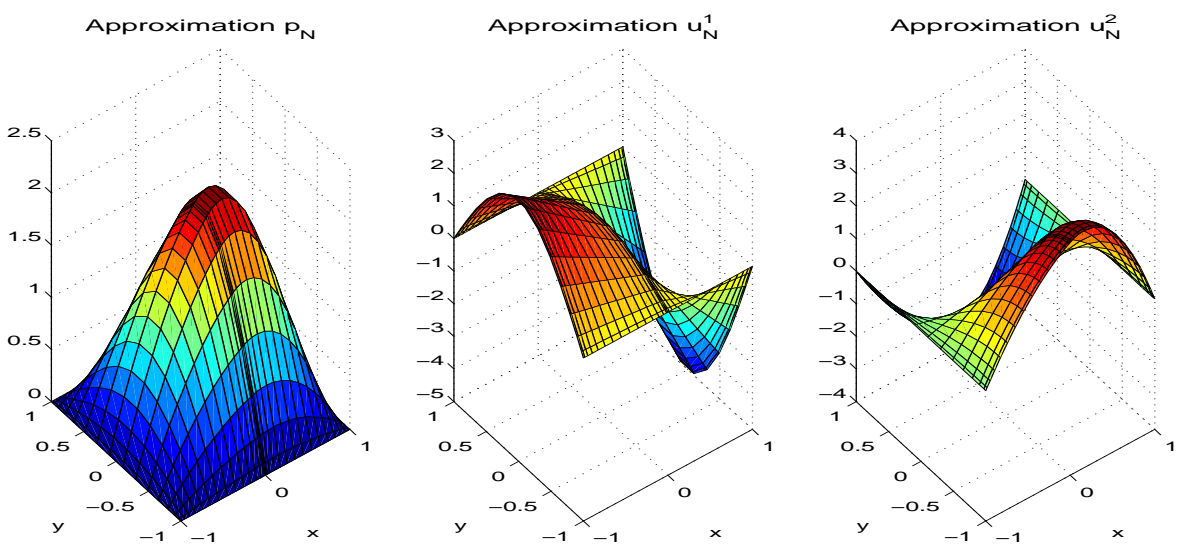

Figure 2. Legendre approximation of $\left[\dot{H}^{1} \times H^{1}\right]$ FOSLS $(\sigma=1)$. 

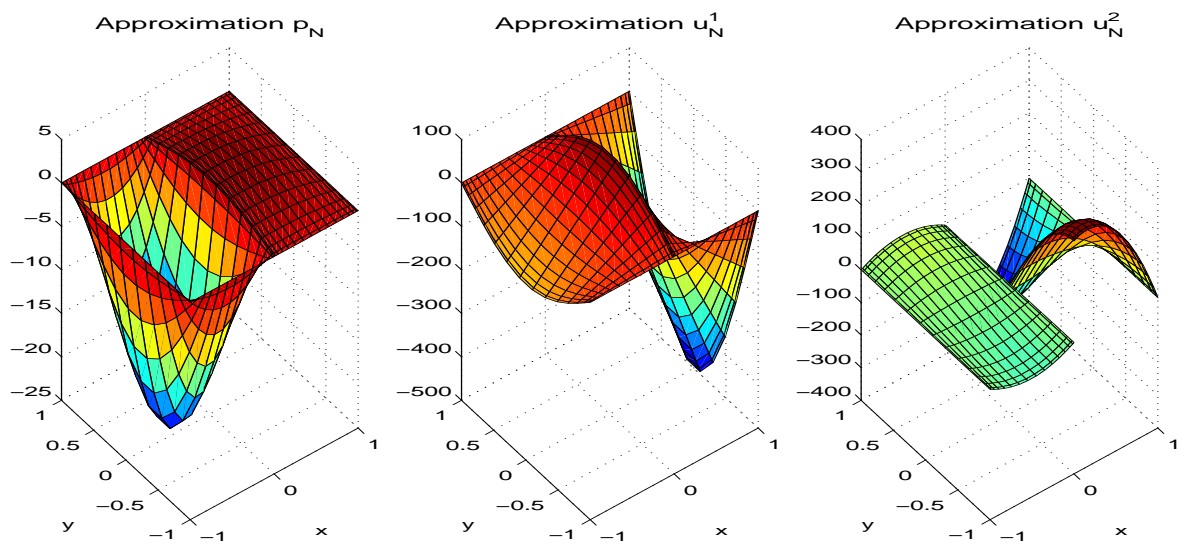

FIGURE 3. Legendre approximation of $\left[\dot{H}^{1} \times H^{1}\right]$ FOSLS $(\sigma=100)$.

Figures 2 and 3 show the numerical solutions for $\sigma=1$ and $\sigma=100$, respectively. One may see that the second component of flux variable $\mathbf{u}$ has a big jump in Figure 2. TABLES 1 through 6 show that the proposed method has spectral convergence in $\left[\dot{H}^{1}(\Omega)\right]^{2} \times H^{1}(\Omega)$-norm of pseudo-spectral Legendre/Chebyshev collocation least-squares method for both variables $p$ and $\mathbf{u}$, regardless of coefficients $a, \mathbf{b}$ and $c_{0}$. One may compare the numerical results with those of the least-squares finite element approximations in [13] and negative norm least-squares pseudo-spectral approximations in [22]. The numerical results show that the pseudo-spectral approximations of both $\left[\dot{H}^{1} \times H^{1}\right]$-FOSLS and $\left[H_{d i v} \times H^{1}\right]$-FOSLS have very good performances similarly. Comparing Legendre approximations in Tables 1 and 4 with Chebyshev approximations in Tables 3 and 6 , the discretization errors for Legendre approximation are a little bit smaller than those of Chebyshev approximation, but the difference seems to be very small.

TABLE 1. Legendre approximation of $\left[\dot{H}^{1} \times H^{1}\right]$ FOSLS $(\sigma=1)$.

\begin{tabular}{c|c|c|cccc}
\hline $\mathbf{b}$ & $c_{0}$ & $N$ & $\left\|e_{p}\right\|_{L^{2}(\Omega)}$ & $\left\|e_{p}\right\|_{\dot{H}^{1}(\Omega)}$ & $\left\|e_{\mathbf{u}}\right\|_{L^{2}(\Omega)}$ & $\left\|e_{\mathbf{u}}\right\|_{\dot{H}^{1}(\Omega)}$ \\
\hline $\mathbf{0}$ & 0 & 3 & $2.035 \mathrm{e}-02$ & $5.016 \mathrm{e}-02$ & $2.978 \mathrm{e}-01$ & $1.654 \mathrm{e}+00$ \\
& & 7 & $8.725 \mathrm{e}-07$ & $7.438 \mathrm{e}-06$ & $1.200 \mathrm{e}-04$ & $1.834 \mathrm{e}-03$ \\
& & 11 & $1.191 \mathrm{e}-11$ & $1.764 \mathrm{e}-10$ & $5.416 \mathrm{e}-09$ & $1.376 \mathrm{e}-07$ \\
& & 15 & $2.259 \mathrm{e}-14$ & $6.507 \mathrm{e}-14$ & $3.743 \mathrm{e}-13$ & $8.796 \mathrm{e}-12$ \\
\hline $\mathbf{0}$ & -10 & 3 & $7.123 \mathrm{e}-02$ & $2.123 \mathrm{e}-01$ & $4.283 \mathrm{e}-01$ & $1.719 \mathrm{e}+00$ \\
& & 7 & $1.287 \mathrm{e}-05$ & $9.123 \mathrm{e}-05$ & $1.212 \mathrm{e}-04$ & $1.793 \mathrm{e}-03$ \\
& & 11 & $1.308 \mathrm{e}-10$ & $1.899 \mathrm{e}-09$ & $5.405 \mathrm{e}-09$ & $1.371 \mathrm{e}-07$ \\
& & 15 & $2.558 \mathrm{e}-13$ & $9.092 \mathrm{e}-13$ & $9.152 \mathrm{e}-13$ & $7.961 \mathrm{e}-12$ \\
\hline$(6,9)$ & 0 & 3 & $5.567 \mathrm{e}-02$ & $2.248 \mathrm{e}-01$ & $3.662 \mathrm{e}-01$ & $1.900 \mathrm{e}+00$ \\
& & 7 & $5.622 \mathrm{e}-06$ & $4.530 \mathrm{e}-05$ & $1.182 \mathrm{e}-04$ & $1.785 \mathrm{e}-03$ \\
& & 11 & $1.411 \mathrm{e}-10$ & $1.556 \mathrm{e}-09$ & $5.544 \mathrm{e}-09$ & $1.478 \mathrm{e}-07$ \\
& & 15 & $7.516 \mathrm{e}-13$ & $2.510 \mathrm{e}-12$ & $2.601 \mathrm{e}-12$ & $2.315 \mathrm{e}-11$ \\
\hline
\end{tabular}


PSEUDO-SPECTRAL LEAST-SQUARES METHOD FOR INTERFACE PROBLEMS1305

TABLE 2. Legendre approximation of $\left[H_{d i v} \times H^{1}\right]$ FOSLS $(\sigma=1)$.

\begin{tabular}{c|c|c|cccc}
\hline $\mathbf{b}$ & $c_{0}$ & $N$ & $\left\|e_{p}\right\|_{L^{2}(\Omega)}$ & $\left\|e_{p}\right\|_{\dot{H}^{1}(\Omega)}$ & $\left\|e_{\mathbf{u}}\right\|_{L^{2}(\Omega)}$ & $\left\|e_{\mathbf{u}}\right\|_{\dot{H}^{1}(\Omega)}$ \\
\hline $\mathbf{0}$ & 0 & 3 & $1.806 \mathrm{e}-02$ & $4.417 \mathrm{e}-02$ & $2.414 \mathrm{e}-01$ & $9.662 \mathrm{e}-01$ \\
& & 7 & $5.107 \mathrm{e}-07$ & $4.128 \mathrm{e}-06$ & $4.842 \mathrm{e}-05$ & $7.549 \mathrm{e}-04$ \\
& & 11 & $5.227 \mathrm{e}-12$ & $7.104 \mathrm{e}-11$ & $1.482 \mathrm{e}-09$ & $4.896 \mathrm{e}-08$ \\
& & 15 & $2.872 \mathrm{e}-14$ & $7.890 \mathrm{e}-14$ & $4.104 \mathrm{e}-13$ & $1.489 \mathrm{e}-11$ \\
\hline $\mathbf{0}$ & -10 & 3 & $3.892 \mathrm{e}-02$ & $1.044 \mathrm{e}-01$ & $3.126 \mathrm{e}-01$ & $9.789 \mathrm{e}-01$ \\
& & 7 & $6.172 \mathrm{e}-06$ & $4.045 \mathrm{e}-05$ & $5.154 \mathrm{e}-05$ & $7.554 \mathrm{e}-04$ \\
& & 11 & $4.365 \mathrm{e}-11$ & $5.695 \mathrm{e}-10$ & $1.488 \mathrm{e}-09$ & $4.891 \mathrm{e}-08$ \\
& & 15 & $2.056 \mathrm{e}-13$ & $7.366 \mathrm{e}-13$ & $7.382 \mathrm{e}-13$ & $1.619 \mathrm{e}-11$ \\
\hline$(6,9)$ & 0 & 3 & $3.770 \mathrm{e}-02$ & $1.678 \mathrm{e}-01$ & $3.818 \mathrm{e}-01$ & $1.851 \mathrm{e}+00$ \\
& & 7 & $3.164 \mathrm{e}-05$ & $1.141 \mathrm{e}-04$ & $1.652 \mathrm{e}-04$ & $1.835 \mathrm{e}-03$ \\
& & 11 & $9.781 \mathrm{e}-11$ & $9.795 \mathrm{e}-10$ & $4.495 \mathrm{e}-09$ & $1.297 \mathrm{e}-07$ \\
& & 15 & $1.815 \mathrm{e}-13$ & $7.798 \mathrm{e}-13$ & $1.161 \mathrm{e}-12$ & $2.892 \mathrm{e}-11$ \\
\hline
\end{tabular}

TABLE 3. Chebyshev approximation of $\left[\dot{H}^{1} \times H^{1}\right]$ FOSLS $(\sigma=1)$.

\begin{tabular}{c|c|c|cccc}
\hline $\mathbf{b}$ & $c_{0}$ & $N$ & $\left\|e_{p}\right\|_{L^{2}(\Omega)}$ & $\left\|e_{p}\right\|_{\dot{H}^{1}(\Omega)}$ & $\left\|e_{\mathbf{u}}\right\|_{L^{2}(\Omega)}$ & $\left\|e_{\mathbf{u}}\right\|_{\dot{H}^{1}(\Omega)}$ \\
\hline $\mathbf{0}$ & 0 & 3 & $8.839 \mathrm{e}-02$ & $2.921 \mathrm{e}-01$ & $6.130 \mathrm{e}-01$ & $3.657 \mathrm{e}+00$ \\
& & 7 & $1.065 \mathrm{e}-05$ & $1.578 \mathrm{e}-04$ & $2.064 \mathrm{e}-04$ & $3.660 \mathrm{e}-03$ \\
& & 11 & $2.145 \mathrm{e}-10$ & $7.000 \mathrm{e}-09$ & $9.750 \mathrm{e}-09$ & $2.834 \mathrm{e}-07$ \\
& & 15 & $1.194 \mathrm{e}-12$ & $5.879 \mathrm{e}-12$ & $8.825 \mathrm{e}-12$ & $1.586 \mathrm{e}-10$ \\
\hline $\mathbf{0}$ & -10 & 3 & $8.194 \mathrm{e}-02$ & $3.899 \mathrm{e}-01$ & $7.478 \mathrm{e}-01$ & $3.839 \mathrm{e}+00$ \\
& & 7 & $3.087 \mathrm{e}-05$ & $2.472 \mathrm{e}-04$ & $2.171 \mathrm{e}-04$ & $3.545 \mathrm{e}-03$ \\
& & 11 & $3.341 \mathrm{e}-10$ & $8.088 \mathrm{e}-09$ & $9.680 \mathrm{e}-09$ & $2.822 \mathrm{e}-07$ \\
& & 15 & $6.706 \mathrm{e}-12$ & $2.959 \mathrm{e}-11$ & $3.167 \mathrm{e}-11$ & $1.475 \mathrm{e}-10$ \\
\hline$(6,9)$ & 0 & 3 & $1.735 \mathrm{e}-01$ & $5.956 \mathrm{e}-01$ & $6.448 \mathrm{e}-01$ & $3.532 \mathrm{e}+00$ \\
& & 7 & $1.271 \mathrm{e}-05$ & $1.748 \mathrm{e}-04$ & $2.333 \mathrm{e}-04$ & $3.908 \mathrm{e}-03$ \\
& & 11 & $2.879 \mathrm{e}-10$ & $7.591 \mathrm{e}-09$ & $1.051 \mathrm{e}-08$ & $3.175 \mathrm{e}-07$ \\
& & 15 & $1.328 \mathrm{e}-12$ & $9.310 \mathrm{e}-12$ & $1.119 \mathrm{e}-11$ & $1.579 \mathrm{e}-10$ \\
\hline
\end{tabular}

TABLE 4. Legendre approximation of $\left[\dot{H}^{1} \times H^{1}\right]$ FOSLS $(\sigma=100)$.

\begin{tabular}{c|c|c|cccc}
\hline $\mathbf{b}$ & $c_{0}$ & $N$ & $\left\|e_{p}\right\|_{L^{2}(\Omega)}$ & $\left\|e_{p}\right\|_{\dot{H}^{1}(\Omega)}$ & $\left\|e_{\mathbf{u}}\right\|_{L^{2}(\Omega)}$ & $\left\|e_{\mathbf{u}}\right\|_{\dot{H}^{1}(\Omega)}$ \\
\hline$(6,9)$ & 0 & 3 & $2.785 \mathrm{e}-01$ & $1.508 \mathrm{e}+00$ & $2.292 \mathrm{e}+01$ & $1.136 \mathrm{e}+02$ \\
& & 7 & $1.789 \mathrm{e}-04$ & $1.006 \mathrm{e}-03$ & $4.253 \mathrm{e}-03$ & $6.156 \mathrm{e}-02$ \\
& & 11 & $1.732 \mathrm{e}-09$ & $1.928 \mathrm{e}-08$ & $1.289 \mathrm{e}-07$ & $4.208 \mathrm{e}-06$ \\
& & 15 & $5.845 \mathrm{e}-12$ & $2.384 \mathrm{e}-11$ & $7.609 \mathrm{e}-11$ & $8.485 \mathrm{e}-10$ \\
\hline$(6,9)$ & -10 & 3 & $8.181 \mathrm{e}-01$ & $3.628 \mathrm{e}+00$ & $2.293 \mathrm{e}+01$ & $1.130 \mathrm{e}+02$ \\
& & 7 & $7.024 \mathrm{e}-04$ & $3.663 \mathrm{e}-03$ & $7.081 \mathrm{e}-03$ & $6.661 \mathrm{e}-02$ \\
& & 11 & $5.322 \mathrm{e}-09$ & $4.109 \mathrm{e}-08$ & $1.347 \mathrm{e}-07$ & $4.195 \mathrm{e}-06$ \\
& & 15 & $1.928 \mathrm{e}-12$ & $1.060 \mathrm{e}-11$ & $3.683 \mathrm{e}-11$ & $7.908 \mathrm{e}-10$ \\
\hline
\end{tabular}


TABLE 5. Legendre approximation of $\left[H_{d i v} \times H^{1}\right]$ FOSLS $(\sigma=100)$.

\begin{tabular}{c|c|c|cccc}
\hline $\mathbf{b}$ & $c_{0}$ & $N$ & $\left\|e_{p}\right\|_{L^{2}(\Omega)}$ & $\left\|e_{p}\right\|_{\dot{H}^{1}(\Omega)}$ & $\left\|e_{\mathbf{u}}\right\|_{L^{2}(\Omega)}$ & $\left\|e_{\mathbf{u}}\right\|_{\dot{H}^{1}(\Omega)}$ \\
\hline$(6,9)$ & 0 & 3 & $3.397 \mathrm{e}+00$ & $1.410 \mathrm{e}+01$ & $5.257 \mathrm{e}+01$ & $2.020 \mathrm{e}+02$ \\
& & 7 & $3.343 \mathrm{e}-04$ & $3.341 \mathrm{e}-03$ & $1.400 \mathrm{e}-02$ & $1.884 \mathrm{e}-01$ \\
& & 11 & $3.613 \mathrm{e}-09$ & $6.489 \mathrm{e}-08$ & $4.222 \mathrm{e}-07$ & $1.120 \mathrm{e}-05$ \\
& & 15 & $1.247 \mathrm{e}-11$ & $5.684 \mathrm{e}-11$ & $1.796 \mathrm{e}-10$ & $1.465 \mathrm{e}-09$ \\
\hline$(6,9)$ & -10 & 3 & $6.141 \mathrm{e}+00$ & $2.523 \mathrm{e}+01$ & $7.534 \mathrm{e}+01$ & $2.640 \mathrm{e}+02$ \\
& & 7 & $7.475 \mathrm{e}-04$ & $4.987 \mathrm{e}-03$ & $1.541 \mathrm{e}-02$ & $1.956 \mathrm{e}-01$ \\
& & 11 & $4.031 \mathrm{e}-09$ & $7.097 \mathrm{e}-08$ & $4.250 \mathrm{e}-07$ & $1.122 \mathrm{e}-05$ \\
& & 15 & $3.787 \mathrm{e}-11$ & $1.672 \mathrm{e}-10$ & $4.180 \mathrm{e}-10$ & $2.231 \mathrm{e}-09$ \\
\hline
\end{tabular}

TABLE 6. Chebyshev approximation of $\left[H_{d i v} \times H^{1}\right]$ FOSLS $(\sigma=100)$.

\begin{tabular}{c|c|c|cccc}
\hline $\mathbf{b}$ & $c_{0}$ & $N$ & $\left\|e_{p}\right\|_{L^{2}(\Omega)}$ & $\left\|e_{p}\right\|_{\dot{H}^{1}(\Omega)}$ & $\left\|e_{\mathbf{u}}\right\|_{L^{2}(\Omega)}$ & $\left\|e_{\mathbf{u}}\right\|_{\dot{H}^{1}(\Omega)}$ \\
\hline$(6,9)$ & 0 & 3 & $5.690 \mathrm{e}+00$ & $3.388 \mathrm{e}+01$ & $1.066 \mathrm{e}+02$ & $4.086 \mathrm{e}+02$ \\
& & 7 & $9.437 \mathrm{e}-04$ & $1.408 \mathrm{e}-02$ & $3.386 \mathrm{e}-02$ & $4.948 \mathrm{e}-01$ \\
& & 11 & $1.129 \mathrm{e}-08$ & $3.519 \mathrm{e}-07$ & $1.104 \mathrm{e}-06$ & $3.374 \mathrm{e}-05$ \\
& & 15 & $2.492 \mathrm{e}-10$ & $1.967 \mathrm{e}-09$ & $5.490 \mathrm{e}-09$ & $2.981 \mathrm{e}-08$ \\
\hline$(6,9)$ & -10 & 3 & $8.257 \mathrm{e}+00$ & $4.890 \mathrm{e}+01$ & $1.399 \mathrm{e}+02$ & $5.056 \mathrm{e}+02$ \\
& & 7 & $2.161 \mathrm{e}-03$ & $2.202 \mathrm{e}-02$ & $4.509 \mathrm{e}-02$ & $5.592 \mathrm{e}-01$ \\
& & 11 & $1.353 \mathrm{e}-08$ & $3.691 \mathrm{e}-07$ & $1.121 \mathrm{e}-06$ & $3.381 \mathrm{e}-05$ \\
& & 15 & $1.052 \mathrm{e}-09$ & $7.688 \mathrm{e}-09$ & $1.888 \mathrm{e}-08$ & $8.210 \mathrm{e}-08$ \\
\hline
\end{tabular}

Example 2 (Discontinuous diffusion coefficient with a singular source term). Let $\Omega=(0, L) \times(0,1)$ and $\Gamma=\{\alpha\} \times(0,1)$. Consider the following elliptic problem with the singular source term:

$$
\begin{aligned}
-a p_{x x}-p_{y y}+u & =f+\nu \delta_{\alpha}(x) \sin \pi y \text { in } \Omega, \\
p & =0 \text { on } \partial \Omega,
\end{aligned}
$$

with the following exact solution

$$
p(x, y)=\sin \pi y \cdot \begin{cases}C_{1} \cos \left(x \gamma_{1}\right)+C_{2} \sin \left(x \gamma_{1}\right)+1, & x \in(0, \alpha), \\ C_{3} \cos \left(x \gamma_{2}\right)+C_{4} \sin \left(x \gamma_{2}\right)+1, & x \in(\alpha, L),\end{cases}
$$

where

$$
\left\{\begin{array} { c } 
{ a _ { 1 } = 1 0 0 , } \\
{ a _ { 2 } = 1 0 , }
\end{array} \quad \left\{\begin{array} { c } 
{ \gamma _ { 1 } = 1 / 1 0 , } \\
{ \gamma _ { 2 } = 1 / \sqrt { 1 0 } , }
\end{array} \quad \left\{\begin{array}{l}
\alpha=\sqrt{10} \pi / 6, \\
L=\sqrt{10} \pi / 2 .
\end{array}\right.\right.\right.
$$

In this example, $C_{i}$ can be determined by the homogeneous boundary conditions and the jump conditions, $a_{1} \partial_{x} p-a_{2} \partial_{x} p=\nu \sin \pi y$ on $\Gamma$, and the right hand side $f$ can also be given by easy computation of the left hand side with solution $p$. With two sub-domains, $\Omega_{1}=(0, \alpha) \times(0,1)$ and $\Omega_{2}=(\alpha, L) \times(0,1)$, we applied least-squares pseudo-spectral collocation method to solve the above singular problem as an interface problem.

Figures 4 and 5 show the numerical solutions for $\nu=5$ and $\nu=-5$, respectively. One may see that the first component of flux variable $\mathbf{u}$ has big jumps 
in Figures 4 and 5. TABLES 7 through 10 show that we have the spectral convergence in $\left[\dot{H}^{1}(\Omega)\right]^{2} \times H^{1}(\Omega)$-norm of pseudo-spectral Legendre/Chebyshev collocation least-squares method for both variables $p$ and $\mathbf{u}$, regardless of $\nu$. The tables also show that the numerical results are very similar for both $\left[\dot{H}^{1} \times H^{1}\right]$-FOSLS and $\left[H_{d i v} \times H^{1}\right]$-FOSLS. Comparing Legendre approximation with Chebyshev approximation, the discretization errors for Legendre approximation are a little bit smaller than those of Chebyshev approximation like the previous example.
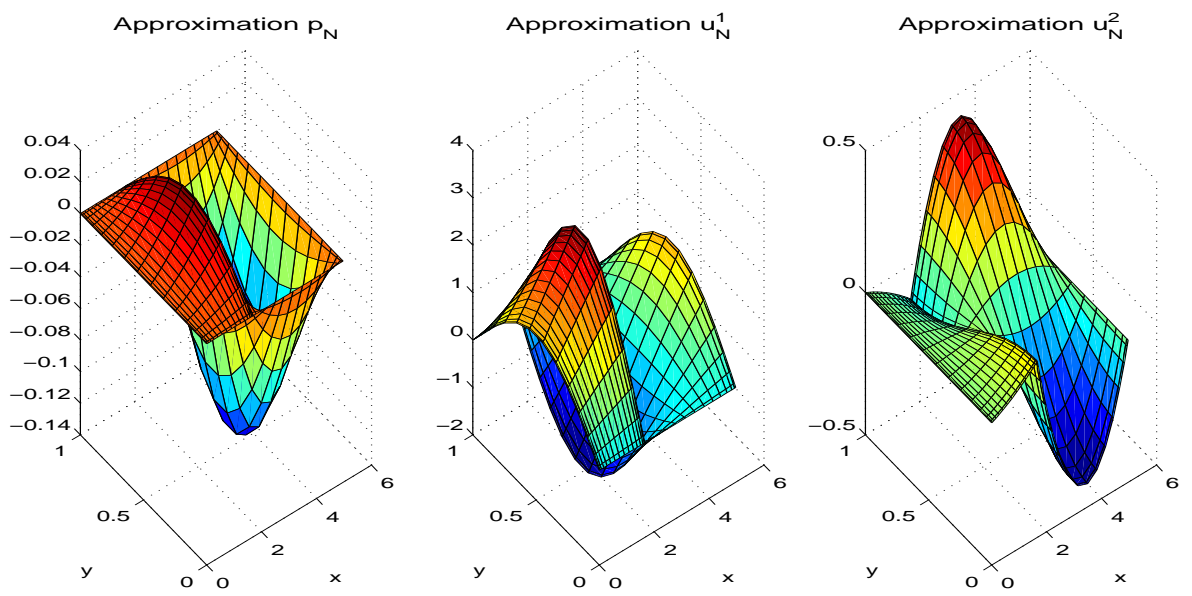

FiguRE 4. Legendre approximation of $\left[\dot{H}^{1} \times H^{1}\right]$ FOSLS $(\nu=5)$.
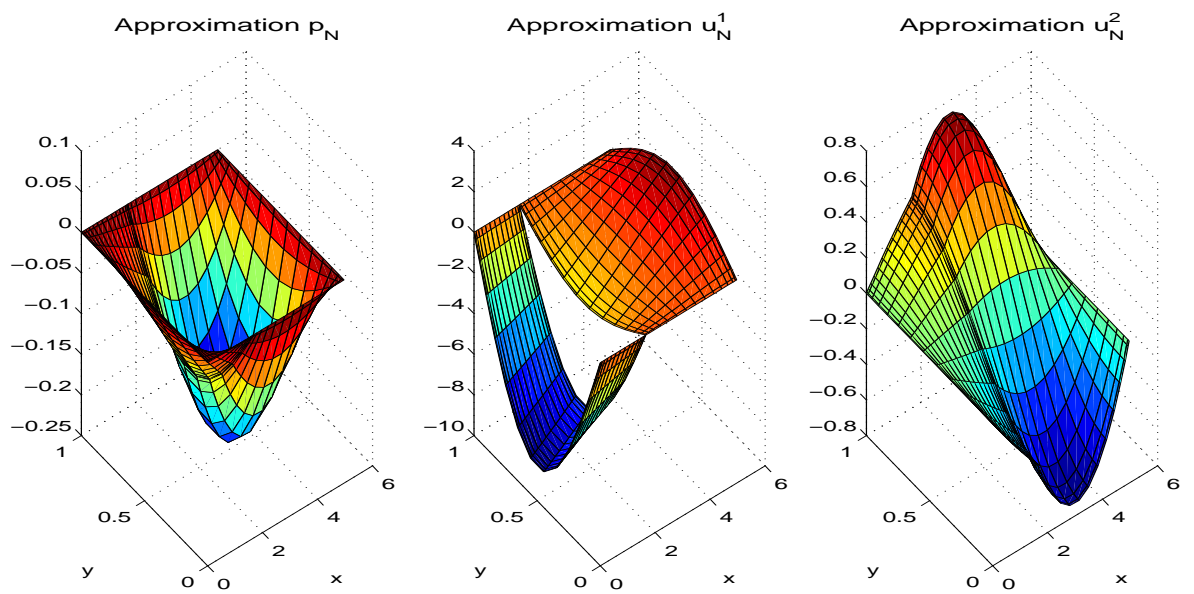

FigURE 5. Legendre approximation of $\left[\dot{H}^{1} \times H^{1}\right]$ FOSLS $(\nu=-5)$. 
TABLE 7. Legendre approximation of $\left[\dot{H}^{1} \times H^{1}\right]$ FOSLS.

\begin{tabular}{l|c|cccc}
\hline$\nu$ & $N$ & $\left\|e_{p}\right\|_{L^{2}(\Omega)}$ & $\left\|e_{p}\right\|_{\dot{H}^{1}(\Omega)}$ & $\left\|e_{\mathbf{u}}\right\|_{L^{2}(\Omega)}$ & $\left\|e_{\mathbf{u}}\right\|_{\dot{H}^{1}(\Omega)}$ \\
\hline 5 & 2 & $4.232 \mathrm{e}-02$ & $1.311 \mathrm{e}-01$ & $6.361 \mathrm{e}-01$ & $2.472 \mathrm{e}+00$ \\
& 6 & $9.690 \mathrm{e}-06$ & $1.088 \mathrm{e}-04$ & $2.664 \mathrm{e}-04$ & $3.728 \mathrm{e}-03$ \\
& 10 & $2.267 \mathrm{e}-10$ & $4.597 \mathrm{e}-09$ & $1.519 \mathrm{e}-08$ & $3.783 \mathrm{e}-07$ \\
& 14 & $8.847 \mathrm{e}-15$ & $1.135 \mathrm{e}-13$ & $6.599 \mathrm{e}-13$ & $1.162 \mathrm{e}-11$ \\
\hline-5 & 2 & $1.035 \mathrm{e}-01$ & $3.196 \mathrm{e}-01$ & $1.942 \mathrm{e}+00$ & $7.013 \mathrm{e}+00$ \\
& 6 & $2.473 \mathrm{e}-05$ & $2.959 \mathrm{e}-04$ & $1.169 \mathrm{e}-03$ & $1.655 \mathrm{e}-02$ \\
& 10 & $7.953 \mathrm{e}-10$ & $1.704 \mathrm{e}-08$ & $6.366 \mathrm{e}-08$ & $1.503 \mathrm{e}-06$ \\
& 14 & $2.835 \mathrm{e}-14$ & $4.628 \mathrm{e}-13$ & $2.425 \mathrm{e}-12$ & $5.178 \mathrm{e}-11$ \\
\hline
\end{tabular}

TABLE 8. Legendre approximation of $\left[H_{d i v} \times H^{1}\right]$ FOSLS.

\begin{tabular}{l|c|cccc}
\hline$\nu$ & $N$ & $\left\|e_{p}\right\|_{L^{2}(\Omega)}$ & $\left\|e_{p}\right\|_{\dot{H}^{1}(\Omega)}$ & $\left\|e_{\mathbf{u}}\right\|_{L^{2}(\Omega)}$ & $\left\|e_{\mathbf{u}}\right\|_{\dot{H}^{1}(\Omega)}$ \\
\hline 5 & 2 & $5.072 \mathrm{e}-02$ & $1.572 \mathrm{e}-01$ & $7.268 \mathrm{e}-01$ & $2.825 \mathrm{e}+00$ \\
& 6 & $1.552 \mathrm{e}-05$ & $2.013 \mathrm{e}-04$ & $5.343 \mathrm{e}-04$ & $1.052 \mathrm{e}-02$ \\
& 10 & $4.649 \mathrm{e}-10$ & $1.027 \mathrm{e}-08$ & $3.726 \mathrm{e}-08$ & $1.607 \mathrm{e}-06$ \\
& 14 & $1.046 \mathrm{e}-14$ & $1.651 \mathrm{e}-13$ & $9.781 \mathrm{e}-13$ & $4.445 \mathrm{e}-11$ \\
\hline-5 & 2 & $1.150 \mathrm{e}-01$ & $3.550 \mathrm{e}-01$ & $2.133 \mathrm{e}+00$ & $7.708 \mathrm{e}+00$ \\
& 6 & $3.543 \mathrm{e}-05$ & $4.646 \mathrm{e}-04$ & $1.738 \mathrm{e}-03$ & $2.845 \mathrm{e}-02$ \\
& 10 & $1.344 \mathrm{e}-09$ & $3.024 \mathrm{e}-08$ & $1.149 \mathrm{e}-07$ & $3.772 \mathrm{e}-06$ \\
& 14 & $2.903 \mathrm{e}-14$ & $5.092 \mathrm{e}-13$ & $2.927 \mathrm{e}-12$ & $1.068 \mathrm{e}-10$ \\
\hline
\end{tabular}

TABLE 9. Chebyshev approximation of $\left[\dot{H}^{1} \times H^{1}\right]$ FOSLS.

\begin{tabular}{l|c|cccc}
\hline$\nu$ & $N$ & $\left\|e_{p}\right\|_{L^{2}(\Omega)}$ & $\left\|e_{p}\right\|_{\dot{H}^{1}(\Omega)}$ & $\left\|e_{\mathbf{u}}\right\|_{L^{2}(\Omega)}$ & $\left\|e_{\mathbf{u}}\right\|_{\dot{H}^{1}(\Omega)}$ \\
\hline 5 & 2 & $2.083 \mathrm{e}-02$ & $8.893 \mathrm{e}-02$ & $4.611 \mathrm{e}-01$ & $2.430 \mathrm{e}+00$ \\
& 6 & $1.361 \mathrm{e}-05$ & $1.404 \mathrm{e}-04$ & $4.404 \mathrm{e}-04$ & $6.128 \mathrm{e}-03$ \\
& 10 & $3.797 \mathrm{e}-10$ & $7.757 \mathrm{e}-09$ & $2.901 \mathrm{e}-08$ & $7.820 \mathrm{e}-07$ \\
& 14 & $8.215 \mathrm{e}-14$ & $9.542 \mathrm{e}-13$ & $5.717 \mathrm{e}-12$ & $9.221 \mathrm{e}-11$ \\
\hline-5 & 2 & $8.653 \mathrm{e}-02$ & $3.669 \mathrm{e}-01$ & $2.185 \mathrm{e}+00$ & $1.042 \mathrm{e}+01$ \\
& 6 & $3.888 \mathrm{e}-05$ & $4.320 \mathrm{e}-04$ & $1.770 \mathrm{e}-03$ & $2.451 \mathrm{e}-02$ \\
& 10 & $1.404 \mathrm{e}-09$ & $2.898 \mathrm{e}-08$ & $1.002 \mathrm{e}-07$ & $2.380 \mathrm{e}-06$ \\
& 14 & $2.380 \mathrm{e}-13$ & $2.722 \mathrm{e}-12$ & $2.143 \mathrm{e}-11$ & $3.236 \mathrm{e}-10$ \\
\hline
\end{tabular}

TABLE 10. Chebyshev approximation of $\left[H_{d i v} \times H^{1}\right]$ FOSLS.

\begin{tabular}{l|c|cccc}
\hline$\nu$ & $N$ & $\left\|e_{p}\right\|_{L^{2}(\Omega)}$ & $\left\|e_{p}\right\|_{\dot{H}^{1}(\Omega)}$ & $\left\|e_{\mathbf{u}}\right\|_{L^{2}(\Omega)}$ & $\left\|e_{\mathbf{u}}\right\|_{\dot{H}^{1}(\Omega)}$ \\
\hline 5 & 2 & $3.634 \mathrm{e}-02$ & $1.551 \mathrm{e}-01$ & $7.181 \mathrm{e}-01$ & $3.656 \mathrm{e}+00$ \\
& 6 & $2.751 \mathrm{e}-05$ & $3.225 \mathrm{e}-04$ & $9.054 \mathrm{e}-04$ & $1.967 \mathrm{e}-02$ \\
& 10 & $8.603 \mathrm{e}-10$ & $1.855 \mathrm{e}-08$ & $5.990 \mathrm{e}-08$ & $3.014 \mathrm{e}-06$ \\
& 14 & $6.908 \mathrm{e}-14$ & $1.041 \mathrm{e}-12$ & $6.873 \mathrm{e}-12$ & $1.787 \mathrm{e}-10$ \\
\hline-5 & 2 & $1.116 \mathrm{e}-01$ & $4.735 \mathrm{e}-01$ & $2.615 \mathrm{e}+00$ & $1.253 \mathrm{e}+01$ \\
& 6 & $6.338 \mathrm{e}-05$ & $7.709 \mathrm{e}-04$ & $2.757 \mathrm{e}-03$ & $4.894 \mathrm{e}-02$ \\
& 10 & $2.448 \mathrm{e}-09$ & $5.247 \mathrm{e}-08$ & $1.732 \mathrm{e}-07$ & $6.706 \mathrm{e}-06$ \\
& 14 & $2.725 \mathrm{e}-13$ & $2.744 \mathrm{e}-12$ & $1.973 \mathrm{e}-11$ & $5.217 \mathrm{e}-10$ \\
\hline
\end{tabular}




\section{Conclusion}

This paper develops least-squares pseudo-spectral collocation methods for elliptic boundary value problems (1.1) having interface conditions given by discontinuous coefficients and singular source term. From the discontinuity of coefficients and singular source term, we derived the interface conditions and then we imposed such interface conditions to solution spaces. Over such solution spaces, we define two types of continuous least-squares functionals using $\dot{L}^{2}(\Omega)$-norm, so-called 'broken $L^{2}(\Omega)$-norm'. We also define two types of discrete least-squares functionals using discontinuous spectral norm over two sub-domains. In this paper, we have shown that both types of the homogeneous continuous and discrete least-squares functionals are equivalent to appropriate product norms and the proposed methods have the spectral convergence in the broken norms. Finally, we presented some numerical results to provide evidences for analysis and spectral convergence of our proposed methods. The techniques given in this paper can be further combined with the finite element method and spectral element method. It can be also applied to more general interface problems.

\section{References}

[1] A. K. Aziz, R. B. Kellogg, and A. B. Stephens, Least squares methods for elliptic systems, Math. Comp. 44 (1985), no. 169, 53-70.

[2] C. Bernardi and Y. Maday, Approximations spectrales de problèmes aux limites elliptiques, vol. 10 of Mathématiques \& Applications (Berlin) [Mathematics \& Applications], Springer-Verlag, Paris, 1992.

[3] M. Berndt, T. A. Manteuffel, and S. F. McCormick, Analysis of first-order system least squares (FOSLS) for elliptic problems with discontinuous coefficients. II, SIAM J. Numer. Anal. 43 (2005), no. 1, 409-436 (electronic).

[4] M. Berndt, T. A. Manteuffel, S. F. McCormick, and G. Starke, Analysis of first-order system least squares (FOSLS) for elliptic problems with discontinuous coefficients. I, SIAM J. Numer. Anal. 43 (2005), no. 1, 386-408.

[5] P. B. Bochev and M. D. Gunzburger, Analysis of least squares finite element methods for the Stokes equations, Math. Comp. 63 (1994), no. 208, 479-506.

[6] _ Finite element methods of least-squares type, SIAM Rev. 40 (1998), no. 4, 789-837.

[7] P. Boomkamp, B. Boersma, R. Miesen, and G. Beijnon, A chebyshev collocation method for solving two-phase flow stability problems, J. Comput. Phys. 132 (1997), 191-200.

[8] J. H. Bramble, R. D. Lazarov, and J. E. Pasciak, A least-squares approach based on a discrete minus one inner product for first order systems, Math. Comp. 66 (1997), no. 219, 935-955.

[9] Z. Cai, R. Lazarov, T. A. Manteuffel, and S. F. McCormick, First-order system least squares for second-order partial differential equations. I, SIAM J. Numer. Anal. 31 (1994), no. 6, 1785-1799.

[10] Z. Cai, T. A. Manteuffel, and S. F. McCormick, First-order system least squares for second-order partial differential equations. II, SIAM J. Numer. Anal. 34 (1997), no. 2, $425-454$.

[11] Z. Cai and B. C. Shin, The discrete first-order system least squares: the second-order elliptic boundary value problem, SIAM J. Numer. Anal. 40 (2002), no. 1, 307-318 (electronic). 
[12] C. Canuto, M. Y. Hussaini, A. Quarteroni, and T. A. Zang, Spectral Methods in Fluid Dynamics, Springer Series in Computational Physics, Springer-Verlag, New York, 1988.

[13] Y. Cao and M. D. Gunzburger, Least-squares finite element approximations to solutions of interface problems, SIAM J. Numer. Anal. 35 (1998), no. 1, 393-405 (electronic).

[14] G. J. Fix, M. D. Gunzburger, and R. A. Nicolaides, On finite element methods of the least squares type, Comput. Math. Appl. 5 (1979), no. 2, 87-98.

[15] G. J. Fix and E. Stephan, On the finite element-least squares approximation to higher order elliptic systems, Arch. Rational Mech. Anal. 91 (1985), no. 2, 137-151.

[16] D. Funaro, A variational formulation for the Chebyshev pseudospectral approximation of Neumann problems, SIAM J. Numer. Anal. 27 (1990), no. 3, 695-703.

[17] D. Jesperson, A least squares decomposition method for solving elliptic equations, Math. Comp. 31 (1977), no. 140, 873-880.

[18] B.-N. Jiang, The Least-Squares Finite Element Method, Springer-Verlag, Berlin, 1998.

[19] J.-H. Jung, A note on the spectral collocation approximation of some differential equations with singular source terms, J. Sci. Comput. 39 (2009), no. 1, 49-66.

[20] S. D. Kim, H.-C. Lee, and B. C. Shin, Pseudospectral least-squares method for the second-order elliptic boundary value problem, SIAM J. Numer. Anal. 41 (2003), no. 4, 1370-1387 (electronic).

[21] L Least-squares spectral collocation method for the Stokes equations, Numer. Methods Partial Differential Equations 20 (2004), no. 1, 128-139.

[22] S. D. Kim and B. C. Shin, Chebyshev weighted norm least-squares spectral methods for the elliptic problem, J. Comput. Math. 24 (2006), no. 4, 451-462.

[23] A. Loubenets, T. Ali, and M. Hanke, Highly accurate finite element method for onedimensional elliptic interface problems, Appl. Numer. Math. 59 (2009), no. 1, 119-134.

[24] A. I. Pehlivanov, G. F. Carey, and R. D. Lazarov, Least-squares mixed finite elements for second-order elliptic problems, SIAM J. Numer. Anal. 31 (1994), no. 5, 1368-1377.

[25] M. M. J. Proot and M. I. Gerritsma, A least-squares spectral element formulation for the Stokes problem, J. Sci. Comput. 17 (2002), no. 1-4, 285-296.

[26] A. Quarteroni and A. Valli, Numerical Approximation of Partial Differential Equations, vol. 23 of Springer Series in Computational Mathematics, Springer-Verlag, Berlin, 1994.

[27] Z. G. Seftel, A general theory of boundary value problems for elliptic systems with discontinuous coefficients, Ukrain. Mat. Z. 18 (1966), no. 3, 132-136.

[28] B.-C. Shin and J.-H. Jung, Spectral collocation and radial basis function methods for one-dimensional interface problems, Appl. Numer. Math. 61 (2011), no. 8, 911-928.

[29] A.-K. Tornberg and B. Engquist, Numerical approximations of singular source terms in differential equations, J. Comput. Phys. 200 (2004), no. 2, 462-488.

Department of Mathematics

Chonnam National University

GWANGJU 500-757, Korea

E-mail address: bcshin@jnu.ac.kr 